\title{
Visceral Heterotopy, Embodiment and Consanguine: The Gastric Saga Anubha Bajaj*
}

LT Group Company (Daawat Basmati Rice), LTFoods Ltd., Secunderabad, Telangana, India

*Corresponding author: Anubha B, Deputy General Manager, LT Group Company (Daawat Basmati Rice), LTFoods Ltd., Secunderabad, Telangana, India, Tel: 91-9811693956; E-mail: anubha.bajaj@gmail.com

Received Date: February 16, 2018; Accepted Date: February 28, 2018; Published Date: March 8, 2018

Copyright: () 2018 Anubha B. This is an open-access article distributed under the terms of the Creative Commons Attribution License, which permits unrestricted use, distribution, and reproduction in any medium, provided the original author and source are credited.

\begin{abstract}
Heterotopic or ectopic mucosa is ongenital anomaly elucidated as the inhabitance of tissue outside its habitual locale. This tissue is discerned coincidentally and may be asymptomatic or with nonspecific gastrointestinal symptoms. Two categories of heterotopic tissue predominantly crop up in the gastrointestinal tract, PANCREATIC and GASTRIC. Isolated heterotopic gastric mucosa (HGM) can arise at all position's, anywhere along the gastrointestinal tract, from the oesophagus to the rectum. The incidence is sporadic but should be deliberated upon in the differential diagnosis of unexplained pain of the abdomen, bleeding from the gastrointestinal tract or weight loss. Once heterotopia is identified, suitable treatment can be instituted thereby diminishing the danger of complications. Heterotopic or Ectopic tissue is a congenital anomaly, attributed to abnormal embryologic development, of the existence of tissue apart from the typical location, with no neural, vascular, anatomic relation with the main body of an organ in which it customarily exists. Heterotopic gastric mucosa (HGM) was initially detailed by Ewell and Jackson in 1939. The congenital anomaly can be emulated by acquired transition (e.g. pyloric epithelial lining) in some organs e.g. lower oesophagus, duodenal bulb and gall bladder. HGM can appear at any location in the gastrointestinal tract, however, it is exceptional in the lower alimentary canal.
\end{abstract}

Keywords: Heterotopy; Gastrointestinal; Asymptomatic

\section{Introduction}

Approximately the complete oesophagus is afflicted by HGM as an inlet or cervical inlet area in the proximal oesophagus (HGMPE). It is a congenital anomaly which prevails in up to $10 \%$ of the population. Autopsy exhibits up to $70 \%$ extent of HGM in the normal population. A flat, salmon coloured patch is located in the proximal $1 / 3 \mathrm{rd}$ of the oesophagus, distal to upper oesophageal sphincter [1]. The recognition is circumstantial, happening during retraction of the endoscope, done for appraisal of gastrointestinal complaints. Epithelium simulating gastric mucosa of the nonspecialized antral or pyloric type is perceived in inflammatory bowel disease, duodenitis, peptic ulcer etc. and is a scrupulous metaplastic distortion, contingent to chronic inflammation. Elaborate application of fibre optic endoscopy from upper gastrointestinal tract allows the elucidation of HGM (Table 1).

\begin{tabular}{|l|l|l|}
\hline HGM I & Asymptomatic & \\
\hline HGM II & Symptomatic & No morphologic findings \\
\hline HGM III & Symptomatic & Due to morphologic changes \\
\hline HGM IV & Intraepithelial Neoplasia & Malignant transformation \\
\hline HGV V & Cervical oesophageal adenocarcinoma & \\
\hline
\end{tabular}

Table 1: Clinicopathologic classification is founded on clinical, histological and endoscopic paradigm.

The clinical expression can be demarcated into non neoplastic (II and III) in younger patients and neoplastic (IV and V) in the senior community. Acid dependent disease type II and III constitute the conventional laryngo- oesophageal reflux with heartburn, regurgitation, dysphagia, hoarseness, globus sensation, throat irritation, sore throat, chronic cough etc. Non neoplastic variants manifest as ulceration, bleeding, perforation, stricture, tracheaoesophageal fistula, polyps and cysts. Additionally, chronic ear and sinus complexities are outlined. HGMPE elucidates as acute or chronic oesophagitis, when on acid suppression therapy. Gastritis coincides with infection of Helicobacter pylori (a prevalence of 73\%). Abnormal oesophageal motility, nonspecific motor disorder, hypomotility and hypotensive lower oesophageal sphincter are described. Neoplastic conversion in HGPME is unusual. Nonmalignant changes, chronic inflammation, metaplasia, dysphagia are recognized [2].

HGM is isolated in the duodenum except in the duodenal bulb. HGM is correlated with concurrent fundic gland polyps and duodenitis.

HGM is also encountered in the bile ducts impersonating as cholangiocarcinoma. Polypoid lesions are observed in hilar bile ducts. Symptoms of cholecystitis are engendered by HGM perceived in the 
gall bladder. Diarrhoea, fever and mass obstruction of the gall bladder are discerned (Figure 1).

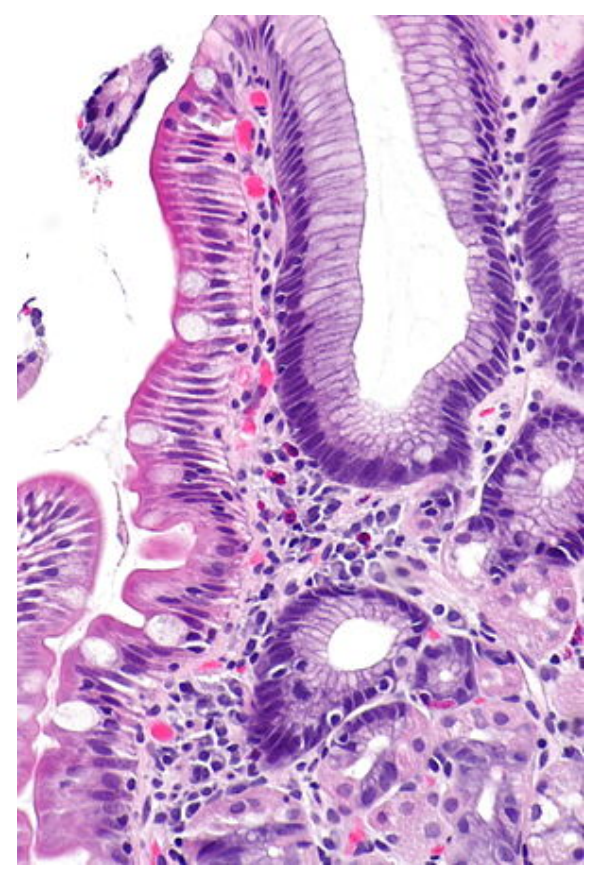

Figure 1: Gastric heterotopy in duodenum.

\section{Histopathology}

The biopsies of HGM are dimunitive, fragmented with complex orientation, displaying well organized group of glands with a combination of parietal and chief cells. Surface epithelium is of a gastric type, blending at the margins with normal duodenal mucosa. Gastric epithelium can be demonstrated by PAS. Small fractions of duodenal mucosa exist with the gastric mucosa. Inflammation is inappreciable. The patient' parameters of age are variable, with distinguishing proximal duodenal endoscopic interpretations, but identical and complementary histopathology [3]. Excellent differentiation and methodical alignment is undoubtedly noticed in congenital mucosal derivatives. Acquired heterotopia symbolizes anomalous regenerative reaction (e.g. pyloric type epithelium in crohn's disease). Therefore, acquired heterotopy is preferably designated as METAPLASIA, comprising of tiny aggregates of parietal or chief cells, central cells or accumulation of fundic glands. Perfectly configured fundic mucosa possibly is the epitome of true congenital heterotopy. Parietal cell heterotopia is consequential to duodenal ulcer or hyperacidity. HGM in the preceding era has been affiliated with 1) Gastric variety of surface epithelium lining the duodenum, a striking aspect of duodenitis and opined as metaplastic. 2) Nodules of pyloric type glands glimpsed in the mucosa, phrased as heteretopic. Parietal or chief cells may be intermittent. These are denoted as metaplastic, the surface change is concomitant to hyperacidity. It frequently prevails in duodenal ulcer. 3) True heterotopy or congenital body type mucosa in the duodenum is an adequately composed mass of flawlessly differentiated body type glands camouflaged with gastric epithelium, reconstituting the integral thickness of duodenal mucosa. Polypoid lesions eventuate with gastric fundic glands and duplication cysts with secondary ulceration and fibrosis. Ectopic tissue ought to be demarcated from gall bladder carcinoma, but ectopic tissue can bolster carcinogenesis. Surgery is essential on account of lack of special clinical and imaging indications [4].

HGM in the large bowel is very exceptional; however it is the most prevalent kind of ectopic tissue in the large bowel. Atypical epithelial differentiation is predisposed by a gene regulator or local inflammation. Heterotopia can ensue from foetal displacement, or an inflammatory response. Manifestations of HGM are painless rectal bleeding with ulceration (50\%) colonic duplication (33\%), perineal ulceration, anal pain, abdominal pain (chronic or colicky), and melena. HGM is discovered as a sequel to investigations for irritable bowel syndrome. HGM is affiliated to polyps succeeded by diverticula, ulcer and reddish appearing mucosal plaques or flaps. MICROSCOPY: HGM of the fundic type, subsequently HGM of the mixed type and the body type (not specified or definable) is the order of appearance of the congenital ectopy of the large gut. Helicobacter Pylori is revealed in rectal HGM. Anorectal HGM may also evolve into neoplasms, benign (adenoma) or malignant (adenocarcinoma) [5]. HGM should be eliminated in the differential diagnosis of rectal bleeding, abdominal pain and proctitis in paediatric patients (Figure 2).

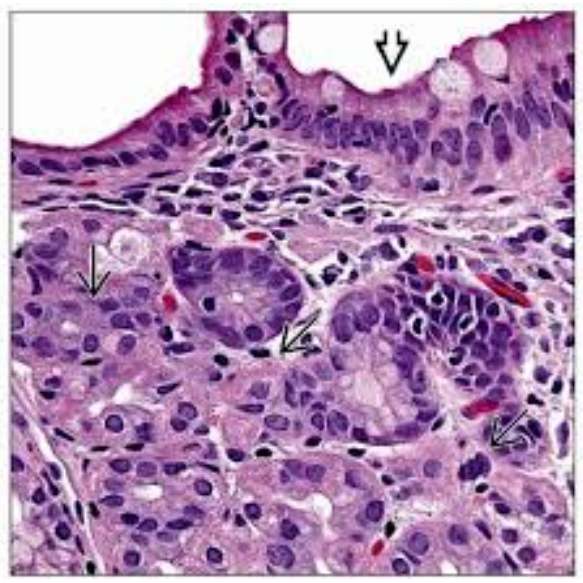

Figure 2: Gastric heterotopy.

\section{Conclusion}

Nonsteroidal anti-inflammatory drugs or other factors encourage ulceration in the stomach. The differential diagnosis in HGM is unexplainable abdominal pain, bleeding from the gastrointestinal tract, weight loss. Appurtenant therapy after ascertainment of HGM curtails the liability of complications.

Congenital heterotopy of the gastric mucosa comprises $2 \%$ of the universal population. The condition has no clinical connotation, no alliance with peptic ulceration. The nodules are miniature and acid/ pepsin secretion is negligible and quickly neutralized.

\section{References}

1. Wlaź J, Mądro A, Kaźmierak W, Celiński K, Słomka M (2014) Pancreatic and Gastric Heterotopy in Gastrointestinal Tract. Postepy Hig Med Dosw (online) 3: 1069-1075.

2. Wollf M (1971) Heterotopic gastric epithelium in the rectum . A report of 3 cases and review of 87 cases of gastric heterotopia in the alimentary canal. Am J Clin Pathol 55: 604-616. 
Citation: Bajaj A (2018) Visceral Heterotopy, Embodiment and Consanguine: The Gastric Saga. Hepatol Pancreat Sci 2: 112. doi:10.4172/ 2573-4563.1000112

Page 3 of 3

3. Hoedemaeker PJ (1970) Hetretopic gastric mucosa in the duodenum. Digestion 3: 165-173.

4. Lessels AM, Martin DF (1982) Heterotopic gastric mucosa in the doudenumJ. Clin. Pathol 35: 591-595.
5. Ewell GH (1939) Aberrant gastric mucosa in the rectum with ulcerations and haemmorrahge. Wis Med J 38: 641-643. 